\title{
Changes and Effects of Dietary Oxidized Lipids in the Gastrointestinal Tract
}

\author{
G. Márquez-Ruiz, M.C. García-Martínez and F. Holgado \\ Instituto del Frío (CSIC), José Antonio Novais 10, 28040 Madrid.
}

\begin{abstract}
This paper is focused on the present state-of-the art of modifications and effects of dietary oxidized lipids during their transit along the gastrointestinal tract. A survey of the literature reporting changes and effects of oxidized lipids before absorption, first in the stomach and then during enzymatic lipolysis in the small intestine, are addressed. Also, the fate of non-absorbed compounds and their potential implications at the colorectal level are discussed. Among the results found, it is shown that acidic gastric conditions and the influence of other dietary components may lead to either further oxidation or antioxidative effects in the stomach. Also, changes in oxidized functions, especially of hydroperoxy and epoxy groups, seem likely to occur. Enzymatic hydrolysis by pancreatic lipase is not effective for triacylglycerol polymers, and hence they can be found as non-absorbed oxidized lipids in the large intestine. Interactions of oxidized lipids with cholesterol absorption in the small intestine and with microflora metabolism have been also observed.
\end{abstract}

Keywords: dietary oxidized lipids, gastrointestinal tract, hydroperoxides, polymers, gastric conditions, nonabsorbed lipids

\section{Introduction}

Lipid oxidation is a major cause of deteriorative changes in foods, especially in relation to sensory properties and concerning the formation of potentially harmful compounds. The oxidation process in food lipids involves a complex series of reactions and gives rise to a high number of different compounds (Frankel, 2005). Lipid oxidation products have attracted much attention because of the wide variety of degenerative processes and diseases associated, including mutagenesis, cell transformation and cancer; atherosclerosis, heart attacks and chronic inflammatory diseases (Cohn, 2002; Kanazawa et al. 2002; Saguy and Dana, 2002; Kanazawa et al. 2002; Dobarganes and Márquez-Ruiz, 2003; Gigun-Sherki et al. 2003, 2004; Spiteller, 2006; Márquez-Ruiz and Dobarganes, 2006; Kanner, 2007). However, it is often difficult to distinguish between the effects of lipid oxidation compounds coming from the diet and of those produced in vivo, the latter depending greatly on the efficiency of defense enzymatic systems.

The gastrointestinal tract is constantly exposed to dietary oxidized lipids and the possible modifications and effects occurring are often ignored (Dobarganes and Márquez-Ruiz, 2003; Márquez-Ruiz and Dobarganes, 2006; Kanner, 2007). It is essential to investigate their chemical changes in the upper gastrointestinal tract in order to know the structure and oxidized functions of molecules bioavailable for absorption in the intestinal lumen and hence for metabolic effects. Also, effects of non-absorbed oxidized lipids along the small and large intestines should not be disregarded because of possible interactions with mucosa and microflora metabolism.

In this review, studies reporting changes and effects of dietary oxidized lipids in the gastrointestinal tract are addressed. Physiological implications of oxidized lipids including their involvement in degenerative processes and diseases upon absorption are, however, beyond the scope of this review. A first part of the paper will include a brief overview on formation and nature of dietary oxidized lipids. Subsequent parts will be dedicated to gastric changes, to events occurring during enzymatic hydrolysis and absorption and lastly to the fate and potential implications of non-absorbed lipids in the large intestine. Figure 1 illustrates the main changes and effects reported.

\section{Dietary Oxidized Lipids: Formation and Nature}

Triacylglycerols are the major constituents of dietary lipids, normally accounting for over 95\% of food lipids, and thereby the most important substrates contributing to oxidized dietary lipids. Specifically,

Correspondence: Gloria Márquez Ruiz, Instituto del Frío (CSIC), José Antonio Novais 10, 28040 Madrid. Tel: +34 915445607; Email: gmarquez@if.csic.es

Copyright in this article, its metadata, and any supplementary data is held by its author or authors. It is published under the Creative Commons Attribution By licence. For further information go to: http://creativecommons.org/licenses/by/3.0/. 


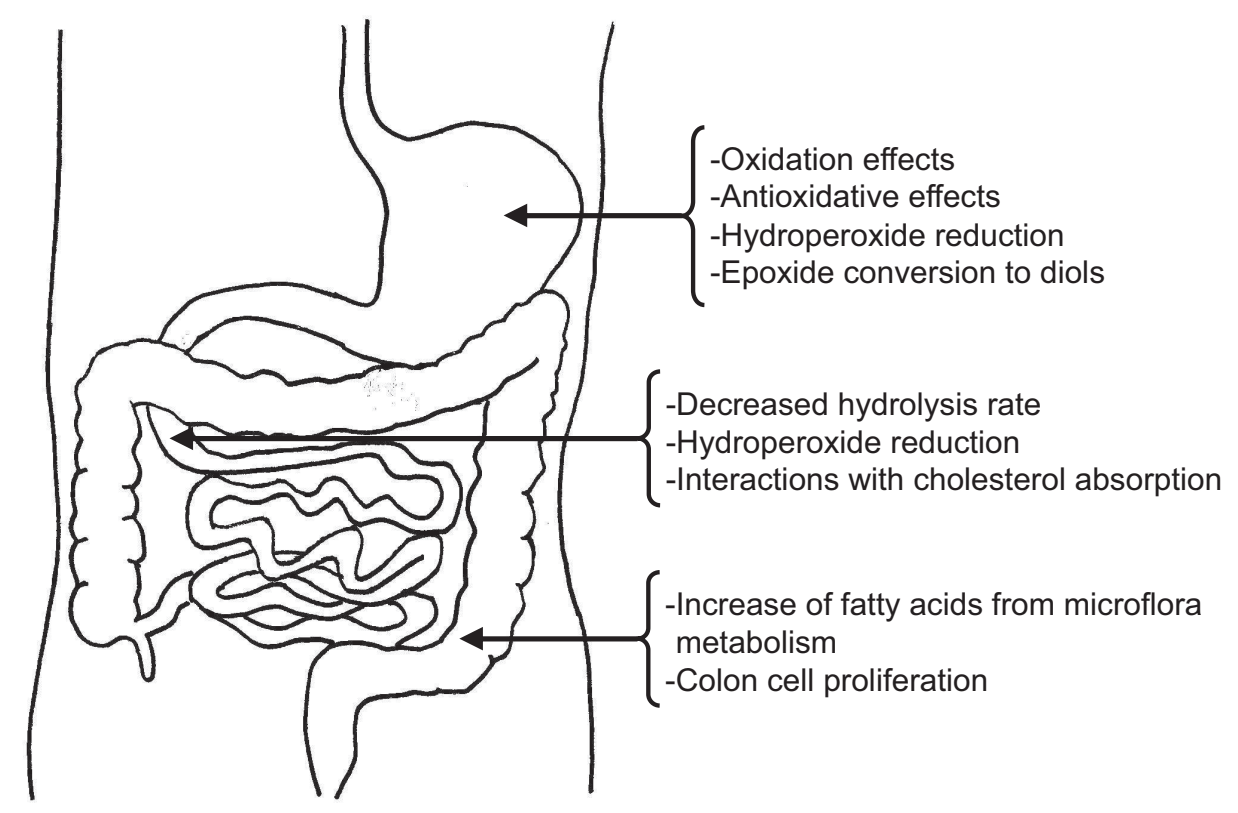

Figure 1. Main changes and effects reported for dietary oxidized lipids in the gastrointestinal tract.

oxidation occurs in the unsaturated fatty acyl groups of triacylglycerols. Oxidation also affects minor lipidic compounds such as phospholipids, sterols, carotenoids, hydrocarbons and phenolic compounds.

The mechanism of lipid autoxidation is wellestablished and involves a catalytic process which proceeds via a free radical mechanism (Frankel, 2005). The initiation step consists on alkyl radical formation in the carbon adjacent to the double bond and the propagation step on addition of oxygen to form alkylperoxyl radicals, hence the oxygen consumed is primarily converted to hydroperoxides. The main mechanism for volatile formation is through $\beta$-scission of the alkoxy radical derived from hydroperoxides. Volatile oxidation compounds are formed in very low amounts and are of the utmost importance from the quality point of view but not from the nutritional point of view. On the other hand, breakdown, decomposition and further reaction of hydroperoxides and the radicals involved result in final oxidation products including oxidized triacylglycerols, dimeric and oligomeric triacylglycerols. Oxidized triacylglycerols include short-chain fatty acyl and short-chain n-oxo fatty acyl groups as well as different oxygenated groups such as hydroxy, keto and epoxy. Formation of dimers and higher polymers is favored at high temperatures such as during frying (Dobarganes and Márquez-Ruiz, 2006; Sanchez-Muniz et al. 2007)
Oxidation compounds in foods are formed during all their processing steps from raw product selection to household or industrial preparation, including commercialization and storage periods. At low or moderate temperatures, solubility of oxygen is high and hydroperoxides are the major products formed. The chemistry of oxidation at high temperatures is much more complex since both thermal and oxidative reactions are simultaneously involved (Dobarganes and Márquez-Ruiz, 2006). As temperature increases, the solubility of oxygen decreases drastically although all the oxidation reactions are accelerated and formation of new compounds is very rapid. Hydroperoxides are practically absent above $150^{\circ} \mathrm{C}$ and secondary oxidized triacylglycerol monomers and polymers are formed since the very early stages of heating (Dobarganes and Márquez-Ruiz, 2003). Whereas in foods subjected to oxidation at low temperature, rarely more than $4 \%-5 \%$ of triacylglycerols would be oxidized (corresponding to about 100 meq $\mathrm{O}_{2} / \mathrm{kg}$ fat, level at which rancid odor is clearly detectable), in the case of frying fats the present official regulations establish the limit of new compounds formed in as high as $25 \%$ (Firestone, 1996) and this level is even considerably surpassed in a significant number of products from fast food outlets (Saguy and Dana, 2002). Hence, most of the oxidized lipids in foods are expected to come from processes involving high temperatures. This is particularly important in the 
case of fried foods produced through discontinuous frying process that may lead to high degradation levels (Saguy and Dana, 2002). Nevertheless, there are no data available on the average intake of oxidized lipids, and the chemical structure and abundance of specific oxidized compounds in the diet are still poorly known (Dobarganes and Márquez-Ruiz, 2003).

\section{Dietary Oxidized Lipids Under Gastric Conditions}

Prior to gastric conditions, dietary lipids are subjected to the action of lingual lipases present in the mouth during mastication of a fat-containing food. Although effects on oxidized lipids have not been studied, the short residence time and low enzyme activity is expected to result in negligible effects in the oral cavity (Hamosh, 1984). However, human parotid saliva was found to reduce arachidonic acid hydroperoxides to its corresponding hydroxy arachidonic acid although only in the presence of glutathione. This effect may be favored in the stomach because of the presence of dietary glutathione and the acidic environment (Terao et al. 1993).

Kanner and coworkers have described the stomach as a "bioreactor" capable of further dietary lipid oxidation or antioxidation (Kanner, 2007). The authors suggest that human gastric fluid, which contains absorbed oxygen and a low $\mathrm{pH}$, may be an excellent medium for enhancing oxidation of lipids in the presence of catalysts found in foods (Kanner and Lapidot, 2001). Thus, in vitro experiments using muscle tissues, metmyoglobin or iron ions, incubated in simulated or human gastric fluid, showed enhanced hydroperoxide formation in samples of linoleic acid or soybean oil, and the crossreaction between free radicals produced cooxidized vitamin $\mathrm{E}, \beta$-carotene and vitamin $\mathrm{C}$ (Lapidot et al. 2005a). However, lipid peroxidation and co-oxidation of the vitamins in stomach medium could be inhibited by red-wine polyphenols (Gorelik et al. 2005). This research group have also determined the effect of $\mathrm{pH}$, estimated the oxygen content in the stomach, and investigated the interaction of selected prooxidants and antioxidants in gastric fluids (Gorelik et al. 2005; Lapidot et al. 2005a, 2005b). Most recently, they reported the dual role of saliva in lipid peroxidation under stomach conditions. It was found that lactoperoxidase increased lipid peroxidation whereas thiocyanate and nitrite reduced it in the presence of reducing compounds. Saliva and polyphenols together protected from co-oxidation of vitamin $\mathrm{E}$ in gastric fluids (Kanner, 2007).

During oxidation of foods at low and moderate temperatures, substantial levels of hydroperoxides may be formed and ingested in the diet, and hence modifications under gastric conditions are important to elucidate to what extent they could be absorbed in their intact form. Moreover, a myriade of deleterious effects have been associated with hydroperoxide reactions such as implication in the atherosclerotic process (Penumetcha et al. 2000; Chisolm and Steinberg, 2000) and degenerative disorders (Bull and Bronstein, 1990; Marnett, 1999; Kanazawa et al. 2000; Spiteller, 2006) but contribution of compounds coming from dietary oxidized lipids is unknown.

Kanazawa and coworkers reported that trilinolein hydroperoxides were hydrolyzed in the rat stomach when administered intragastrically at low doses, giving rise to linoleic acid hydroperoxides and hydroxyls, probably through gastric lipases. At high doses, trilinolein hydroperoxides were mostly retained in the stomach. When linoleic acid hydroperoxide was administered intragastrically, it was converted into secondary oxidation compounds, including hydroxyls, epoxyketones, hexanal and 9-oxononanoic acid, time-dependently (Kanazawa and Ashida, 1998a). The fate of linoleic acid hydroperoxides was further investigated chemically and radiochemically and found to decompose mainly to aldehydes and epoxyketones (Kanazawa and Ashida, 1998b). Hydroperoxide modifications have been also observed under simulated gastric conditions, thus suggesting that acidic gastric environment plays an important role in this reaction. Thus, Terao and coworkers found that most phospholipid hydroperoxides were decomposed during a $2 \mathrm{~h}$ incubation period in a simulated gastric juice (Terao and Fukino, 1993).

The gastrointestinal glutathione peroxidases represent a first line of defense against ingested hydroperoxides (Loguercio and Di Pierro, 1999). They convert hydroperoxides to less reactive hydroxy or aldehydic compounds (Aw, 2005). Complex interaction of such detoxifying enzymes with oxidized lipids have been reported, such as inhibition of glutathione peroxidases by aldehydes (Bosh-Morell et al. 1999) and enhanced absorption of hydroperoxides due to glutathione peroxidase inhibition (Aw, 2005). Glutathione 
regulates the action of glutathione peroxidases and glutathione transferases and is abundantly distributed in the mucosal cells of gastrointestinal tract. Since the amount of glutathione ingested with foods affect greatly its concentration in the gastrointestinal tract (Loguercio and Di Pierro, 1999), composition of diet would determine in part modifications of hydroperoxides by glutathione peroxidases before absorption.

Hydrolysis of epoxy compounds to render the corresponding diols may also occur before absorption, under the acidic conditions in gastric media (Maerker, 1988; Piazza et al. 2003; Giuffrida et al. 2004). This is of great importance because monoepoxy compounds have been recently found to be one of the major oxidized groups formed at frying temperatures (Velasco et al. 2004a, 2004b), accounting for about $25 \%$ of total oxidized fatty acyl groups in real used frying oils at the limit of rejection. Depending on the extent of epoxide conversion to hydroxy function, compounds containing hydroxy groups could be available for absorption in substantial amounts and much higher than those ingested in the diet.

Another type of possible modifications occurring to oxidized lipids in the stomach, particularly to those coming from dietary fried products, refer to depolymerization reactions. Possible breakage of dimers and polymers, the major compounds formed under frying temperatures, could explain the unexpectedly high digestibility values found for such compounds and the detection of nonaltered radiolabeled fatty acids in feces from rats fed diets added thermoxidized radiolabeled linoleic acid (Márquez-Ruiz and Dobarganes, 1995).

\section{Enzymatic Hydrolysis and Absorption of Oxidized Dietary Lipids}

Triacylglycerol monohydroperoxides appear to be hydrolyzed by pancreatic lipase at almost the same degree as its original triacylglycerol (Kanazawa and Ashida, 1998a, 1998b). However, as commented before, previous reactions in the stomach may have converted triscylglycerol hydroperoxides into other oxidized compounds.

Also at the intestinal level, further changes of hydroperoxides have been reported. Muller and coworkers investigated the metabolic fate of radiolabeled 13-hydroperoxyoctaecadienoic acid, 13-hydroxyoctadecanoic acid and linoleic acid in
Caco-2 cell monolayers as a model of the intestinal epithelium and found that radioactivity was recovered for both hydroperoxy and hydroxy compounds in diacylglycerols, phospholipids and cholesterol esterified with oxidized fatty acids, thus concluding that food-born hydroperoxy fatty acids are reduced by the gastrointestinal glutathione peroxidase (Muller et al. 2002).

Recently, molecular-level information of the oxidized triacylglycerol structures present in small intestinal mucosa, adipose tissue and lipoproteins of pigs fed diets with oxidized sunflower oils (peroxide value close to $200 \mathrm{meq} / \mathrm{Kg}$ ) has been reported. The authors did not find triacylglycerol hydroperoxides in tissues or lipoproteins but they detected secondary oxidation products, such as triacylglycerol hydroxides, ketones and epoxides (Suomela et al. 2004, 2005).

In contrast to the results above commented which indicate that hydroperoxide are not likely to be absorbed in their intact form because of previous modifications occurring in the stomach and intestinal epithelium, it has been reported that oxidized lipids in the diet are a significant source of plasma hydroperoxides (Nagatsugawa and Kaneda, 1983; Staprans et al. 1994, 1999; Ursini et al. 1998).

As to effects of hydroperoxides on intestinal mucosa, Kanazawa and coworkers (Kanazawa et al. 1988) examined their potential toxicity by measuring activities of sucrase, maltase, and alkaline phosphatase, which reflect the integrity and function of the brush border membrane. They found that hydroperoxides decreased enzyme activities in jejunum and ileum. On the other hand, incubation of Caco-2 cells with oxidized linoleic acid led to increased apo A-I secretion and steadystate mRNA expression, whose level is negatively correlated with atherosclerotic cardiovascular disease (Rong et al. 2002).

Hydroxy fatty acids are present in dietary oxidized lipids as relatively stable compounds and can also be formed by reduction of hydroperoxides prior to intestinal absorption. Studies on ricinoleic acid, a naturally occurring hydroxy fatty acid, indicated that it is efficiently absorbed from the intestinal tract and deposited in the adipose tissue of rats (Perkins et al. 1961; Kieckebusch et al. 1963; Govind Rao et al. 1969), although no evidence was obtained for the incorporation of ${ }^{14} \mathrm{C}$-hydroxy acids into liver lipids in their intact form (Reber and Draper, 1970). Recently, it has 
been reported that hydroperoxylinoleic acid and hydroxylinoleic acid, obtained enzymatically from linoleic acid, are efficiently absorbed in vitro by Caco-2 intestinal cells (Penumetcha et al. 2000). In humans, dietary hydroxy and epoxy fatty acids incorporated in triacylglycerols were found to be well absorbed (Wilson et al. 2002a, $2002 b$ ). However, differences between monoepoxy and diepoxy fatty acids in the rate of absorption were reported, namely, even though their early rate of absorption appeared similar, diepoxy fatty acids were absorbed much less as time went on (Wilson et al. 2002b).

Other compounds present in dietary oxidized fats and specially in fried products, of higher molecular weight than that of parent triacylglycerols, i.e. triacylglycerol dimers and polymers, are not readily hydrolyzed, as shown by in vitro enzymatic hydrolysis studies on thermally oxidized and used frying oils and fats (Arroyo et al. 1996; MárquezRuiz et al. 1992a, 1998; Sánchez-Muniz et al. 2000). Moreover, deficiencies in dimers and polymers hydrolysis were found to affect significantly the release and hence digestibility of oxidized monomeric and even unoxidized fatty acids (Márquez-Ruiz et al. 1991; 1992a, 1992b, 1992c, 1993; Márquez-Ruiz and Dobarganes, 1995; Sánchez-Muniz et al. 1999; GonzálezMuñoz et al. 2003). Impairment of polymeric triacylglycerol hydrolysis and the subsequent effect on cholesterol micellar solubilization could explain that excretion of endogenous sterols, particularly cholesterol, was markedly higher in rats fed diets containing thermoxidized oils versus nonheated oils (Márquez-Ruiz and Dobarganes, 1992). Similarly, Hochgraf et al. have reported greater cholesterol excretion in rats fed diets with $10 \%$ lipid model preparation containing $60 \%$ highly oxidized linoleic acid and hence presumably rich in polymers (Hochgraf et al. 1997, 2000). In contrast, solubilization and intestinal absorption of cholesterol may be favored by the presence of oxidized lipids readily absorbable, as it occurred when testing hydroperoxylinoleic acid and hydroxylinoleic acid (Penumetcha et al. 2002).

Oxysterols are found in diet in very low amounts but they have attracted much attention, specially oxidized cholesterol, because of their association with atherosclerosis (Brown and Jessup, 1999; Staprans et al. 2000; Guardiola et al. 2002). However, the extent of oxidized cholesterol absorption and, in particular, the influence of concomitant presence of other more abundant dietary oxidized lipids, is not clear.

\section{Non-Absorbed Oxidized Lipids in the Large Intestine}

Non-absorbed oxidized lipids coming from incomplete hydrolysis and/or malabsorption pass from the upper small intestine to the most distal parts of the large intestine but their effects on intestinal mucosa and interactions with colonic microflora are poorly known. Composition of nonabsorbed oxidized lipids is expected to be very complex, including non-hydrolyzed triacylglycerols, mostly of polymeric nature, and non-absorbed fatty acids, along with partial glycerides and minor lipid compounds, most containing different types and number of oxygenated funtions.

In studies determining digestibilities of oxidized monomeric, dimeric, and polymeric fatty acyls groups included in thermoxidized oils, quantitative data was obtained on the occurrence of non-absorbed oxidized lipids, ultimately found in fecal lipids. As a result of comparing levels in diets and in feces, about $20 \%$ of oxidized fatty acid monomers and between 50 and $90 \%$ of dimers and polymers were found in excreted lipids (Márquez-Ruiz et al. 1992b, 1992c; Márquez-Ruiz and Dobarganes, 1995). Among polymeric fatty acids, the lowest digestibilities were found for non-polar dimers $(10.9 \%$ on average), i.e. dimers linked through carbonto-carbon bonds and without any oxygenated function in fatty acyl groups. The low digestibility of non-polar dimers, especially when compared to polar dimers, along with the presence of high free levels in non-absorbed lipids, specifically suggested difficulties during the absorption process (Márquez-Ruiz et al. 1993). When endogenous fatty acids, mostly originating from microflora metabolism, were quantitated in fecal lipids, results showed that their excretion increased as the dietary oil alteration was greater (Márquez-Ruiz and Dobarganes, 1992), thus supporting that high concentrations of poorly digested lipids could have led to intestinal microbial modifications.

Polymers constitute a major fraction in used frying oils and fats, reaching a level as high as $12 \%$ on oil in samples at the limit of rejection (25\% polar compounds) (Firestone, 1996). Their low absorption involves their presence in 
non-negligible amounts throughout the gastrointestinal tract and hence the interest to further study potential effects on epythelial cells and microflora metabolism (Márquez-Ruiz and Dobarganes, 2006). In connection with these aspects, it has been reported that both unabsorbed fats and bile acids secreted in response to a high fat intake might injure the intestinal mucose by their detergent activity, and metabolites of bile acids formed by intestinal bacteria (secondary bile acids) act as tumor promoters (Vonk et al. 1997). Also, the potential contribution of intestinal flora to the production of mutagens from the oxidation of fecal lipids and the effect of vitamin E as a chemopreventive agent have been evaluated (Stone and Papas, 1997; Campbell et al. 2003). Other results suggest that highly oxidizable oils such as fish oil may affect lower intestinal microflora through increasing oxidized lipids in caecum (Kuda et al. 2000). In the case of dietary oxidized lipids, much research work remains to be done. Among the aspects of interest in this context are the effects of oxidized lipids on the number and distribution of colonic bacteria, and the potential hydrolysis and other chemical modifications of oxidized lipids by enzymes associated with colonic bacteria.

Particularly in the case of lipid hydroperoxides, their role in mediating cellular and molecular events in degenerative intestinal disorders, with particular emphasis on colorectal cancer, has deserved special attention. It has been reported that the gut immune system constitutes a possible line of defense, as indicated by both in vitro experiments using 13-hydroperoxylinoleic acid on human colonic lamina propria lymphocites and in vivo assays (Gualde et al. 1983; Elitsur et al. 1990). Lipid hydroperoxides were reported to stimulate cell proliferation and DNA synthesis and to induce ornithine decarboxylase activity, indicating tumor-promoting effects (Bull et al. 1984, 1988; Earles et al. 1991). Studies on human colonic Caco-2 cells have shown that subtoxic levels of hydroperoxides disrupted intestinal redox homeostasis, which contributes to apoptosis (Wang et al. 2000). Complex metabolic effects from chronic exposure to subtoxic levels of hydroperoxides have been reported in rats (Tsunada et al. 2003a, 2003b) as well as effects on the expression of vascular endothelial growth factor in human colorectal tumor cells (Jurek et al. 2005).
Regarding mechanisms responsible for intestinal tumor promotion by dietary oxidized lipids, Bull and coworkers investigated the action of hydroxy oleate and stearate, ricinoleic acid, and the $\alpha-\beta$-unsaturated ketone derived from hydroxyoctadecenoic acid, after intrarectal instillation to rats (Bull et al. 1988). It was concluded that the minimal requirement for stimulation of colon cell proliferation was the presence of an oxidized functionality adjacent to a carbon-carbon double bond. Furthermore, an $\alpha-\beta$-unsaturated ketone was suggested as a likely candidate for the ultimate active form. Production of 2,4-dienone containing fatty acids was later demonstrated during the metabolism of hydroperoxy fatty acids in rat colonic homogenates (Bull and Bronstein, 1990). Other experiments described enzymatic activity that catalyzed the conversion of 13-hydroxyoctadecadienoic acid into 2,4-dienone containing fatty acids. The enzyme activity is widely distributed, with the highest activity in the colon and liver (Earles et al. 1991; Bull et al. 1991). The latest findings on the relationship between dietary lipid oxidation and cancer indicate that the effect of peroxyl radicals on DNA consist mainly on depurination and depyrimidation, and base modifications. Accordingly, a high frequency of mutations can be anticipated and these are thought to contribute to carcinogenesis (Kanazawa et al. 2002). One of the DNA adducts formed by 4-hydroxy2-nonenal has been detected in in healthy human colons (Chung et al. 2000).

Dietary components other than lipids may have a great influence on the apparent adverse effects of the latter, as it has been suggested in the case of consumption of red meat. Thus, a hemoglobin-iron rich diet containing safflower oil led to increased incidence of colon cancer in rats, attributable to the generation of peroxyl radicals from dietary or membrane lipids of intestinal epithelial cells since addition of scavenging antioxidants reduced such an incidence (Sawa et al. 1998). Paradoxically, selective cytotoxic or anti-proliferative effects on tumor cells have been observed for certain polyunsaturated fatty acids, associated with the increase in lipid peroxidation in vitro (Diggle, 2002).

\section{Concluding Remarks}

Chemical changes of dietary oxidized lipid molecules before absorption are essential to gain insight into the nature of compounds which are 
majoritarily absorbed and hence available to exert any biological effect in the circulatory system and target organs. Further research in this context is needed, which would help to distinguish between the effects derived from oxidized compounds coming from the diet and those resulting from compounds produced in vivo, and hence to clarify the real significance of normal intakes of oxidized compounds. Another important issue to be addressed, which has been scarcely studied so far, deals with the implications of non-absorbed oxidized compounds during their passage through the small and large intestines. In this respect, mucosa cell modifications and interactions with microflora metabolism are essential research goals.

\section{Acknowledgements}

This work was supported by Spanish Ministery of Education and Science (Project AGL2007-62922).

\section{References}

Aw, T.Y. 2005. Absorption and lymphatic transport of peroxidized lipids by rat small intestine in vivo: role of mucosal GSH. Toxicol Applied Pharmacol., 204:320-8.

Arroyo, R., Sánchez-Muniz, F.J., Cuesta, C. et al. 1996. Hydrolysis of used frying palm olein and sunflower oil catalyzed by porcine pancreatic lipase. Lipids, 31:1133-9.

Bosh-Morell, F., Flohe, L., Marin, N. et al. 1999. 4-Hydroxynonenal inhibits glutathione peroxidase:protection by glutathione. Free Rad. Biol. Med., 26:1383-7.

Brown, A.J. and Jessup, W. 1999. Oxysterols and atherosclerosis. Atherosclerosis, 142:1-28.

Bull, A.W. and Bronstein, J.C. 1990. Production of unsaturated carbonyl compounds during metabolism of hydroperoxy fatty acids by colonic homogenates. Carcinogenesis, 11:1699-704.

Bull, A.W., Earles, S.M. and Bronstein, J.C. 1991. Metabolism of oxidized linoleic acid: distribution of activity for the enzymatic oxidation of 13-hydroxyoctadecadienoic acid to 13-oxooctadecadienoic acid in rat tissues. Prostaglandins, 41:43-50.

Bull, A.W., Nigro, N.D., Golembieski, W.A. et al. 1984. In vivo stimulation of dna synthesis and induction of ornithine decarboxylase in rat colon by fatty acid hydroperoxides, autoxidation products of unsaturated fatty acids. Cancer Res., 44:4924-8.

Bull, A.W., Nigro, N.D. and Marnett, L.J. 1988. Structural requirements for stimulation of colonic cell proliferation by oxidized fatty acids. Cancer Res., 48:1771-6.

Campbell, S., Stone, W.L., Whaley, S. et al. 2003. Development of gamma $(\gamma)$-tocopherol as a colorectal cancer chemopreventive agent. Crit. Rev. Onc. Hematol., 47:249-59.

Chisolm, G.M. and Steinberg, D. 2000. The oxidative modification hypothesis of atherogenesis: an overview. Free Radic. Biol. Med., 28:1815-26.

Cohn, J. 2002. Oxidized fat in the diet, postprandial lipaemia and cardiovascular disease. Curr. Opin. Lipidol., 13:19-24.

Chung, F.L., Nath, R.G., Ocando, J. et al. 2000. Deoxyguanosine adducts of t-4-hydroxy-2-nonenal are endogenous DNA lesions in rodents and humans: detection and potential sources. Cancer Res., $1507-11$.

Diggle, C.P. 2002. In vitro studies on the relationship between polyunsaturated fatty acids and cancer. Tumour or tissue specific effects? Prog. Lipid. Res., 41:240-53.
Dobarganes, M.C. and Márquez-Ruiz, G. 2003. Oxidized fats in foods. Curr. Opin. Clin. Nutr. Metabol. Care, 6:157-63.

Dobarganes, M.C. and Márquez-Ruiz, G. 2006. Formation and analysis of oxidized monomeric, dimeric and higher oligomeric triglycerides. In: Erickson MD, ed. Deep Frying: Chemistry, Nutrition and Practical Applications. Champaign, IL: AOCS Press 87-110.

Earles, S.M., Bronstein, J.C., Winner, D.L. et al. 1991. Metabolism of oxidized linoleic acid: characterization of 13-hydroxyoctadecadienoic acid dehydrogenase activity from rat colonic tissue. Biochim. Biophys. Acta., 1081:174-80.

Elitsur, Y., Bull, A.W. and Luk, G.D. 1990. Modulation of human colonic lamina propria lymphocite proliferation. Digest Dis. Sci., 35:212-20.

Firestone, D. 1996. Regulation of frying fats and oils. Chemistry, Nutrition and Practical Applications. In: Perkins EG, Erickson MD, ed. Deep Frying. Champaign, IL: AOCS Press 323-34.

Frankel, E.N. 2005. Lipid Oxidation 2nd ed. Dundee (UK): The Oily Press Gilgun-Sherkin, Y., Melaned, E. and Offen, D. 2003. Antioxidant treatment in Alzheimer's disease - Current state. J. Mol. Neuroscience, 21:1-11.

Gilgun-Sherkin, Y., Melaned, E. and Offen, D. 2004. The role of oxidative stress in the pathogenesis of multiple sclerosis: the need for effective antioxidant therapy. J. Neurol., 251:261-8.

Giuffrida, F., Destaillats, F., Robert, F. et al. 2004. Formation and hydrolysis of triacylglycerol and sterol epoxides: role of unsaturated triacylglycerol peroxyl radicals. Free Rad. Biol. Med., 37:104-14.

González-Muñoz, M.J., Bastida, S. and Sánchez-Muniz, J.F. 2003. Shortterm in vivo digestibility assessment of a highly oxidized and polymerized sunflower oil. J. Sci. Food Agric., 83:413-8.

Gorelik, S., Lapidot, T., Shaham, I. et al. 2005. Lipid peroxidation and coupled vitamin oxidation in simulated and human gastric fluid inhibited by dietary polyphenols: health implications. J. Agric. Food Chem., 53:3397-402.

Govind Rao, M.K., Risser, N. and Perkins, E.G. 1969. Incorporation of ricinoleic acid into rat lymph lipids. Proc. Soc. Exp. Biol. Med., 131:1369-72.

Gualde, N., Chable-Rabinovuitch, H., Motta, C. et al. 1983. Hydroperoxyeicosatetraenoic acids. Potent inhibitors of lymphocyte responses. Biochim. Biophys. Acta., 750:429-33.

Guardiola, F., Dutta, P., Codony, R. and Savage, G. 2002. Cholesterol and Phytosterol Oxidation Products: Analysis, Occurrence, and Biological Effects. Champaign, IL: AOCS Press.

Hamosh, M. 1984. Lingual lipase. In: Borgstrom B., Brockman HL ed. Lipases., New York: Elsevier 49-80.

Hochgraf, E., Mokady, S. and Cogan, E. 1997. Dietary oxidized linoleic acid modifies lipid composition of rat liver microsomes and increases their fluidity. J. Nutr., 127:681-6.

Hochgraf, E., Mokady, S. and Cogan, E. 2000. Dietary oxidized linoleic acid enhances liver cholesterol biosynthesis and secretion in rats. J. Nutr. Biochem., 11:176-80.

Jurek, D., Udilova, N., Jozkowicz, A. et al. 2005. Dietary lipid hydroperoxides induce expression of vascular endothelial growth factor (VEGF) in human colorectal tumor cells. FASEB. J., 19:97-9.

Kanazawa, K. and Ashida, H. 1998a. Catabolic fate of dietary trilinoleoylglycerol hydroperoxides in rat gastrointestines. Biochim. Biophys. Acta., 1393:336-48.

Kanazawa, K. and Ashida, H. 1998b. Dietary hydroperoxides of linoleic acid decompose to aldehydes in stomach before being absorbed into the body. Biochim. Biophys. Acta., 1393:349-61.

Kanazawa, A., Ashida, H., Minamoto, S. et al. 1988. The effects of orally administered linoleic acid and its autoxidation products on intestinal mucosa in rat. J. Nutr. Sci. Vitaminol., 34:363-73.

Kanazawa, A., Sawa, T., Akaik, T. et al. 2000. Formation of abasic sites in DNA by t-butyl peroxyl radicals: implication for potent genotoxicity of lipid peroxyl radicals. Cancer Letters, 156:51-5.

Kanazawa, A., Sawa, T., Akaik, T. et al. 2002. Dietary lipid peroxidation products and DNA damage in colon carcinogenesis. Eur. J. Lipid. Sci. Technol., 104:439-47.

Kanner, J. 2007. Dietary advanced lipid oxidation endproducts are risk factors to human health. Mol. Nutr. Food Res., 51:1094-101. 
Kanner, J. and Lapidot, T. 2001. The stomach as a bioreactor: dietary lipid peroxidation in the gastric fluid and the effects of plant-derived antioxidants. Free Radic. Biol. Med., 31:1388-95.

Kieckebusch, W., Griem, W., Czok, G. et al. 1963. Nutrition physiological properties of ricinoleic acid methyl ester. Z Ernaehrungswiss, 93:26-34.

Kuda, T., Enomoto, T., Yano, T. et al. 2000. Cecal environment and TBARS level in mice fed corn oil, beef tallow and menhaden fish oil. J. Nutr. Sci. Vitamin., 46:65-70.

Lapidot, T., Granit, R. and Kanner, J. 2005. Lipid peroxidation by "free" iron ions and myoglobin as affected by dietary antioxidants in simulated gastric fluids. J. Agric. Food Chem., 53:3383-90.

Lapidot, T., Granit, R. and Kanner, J. 2005. Lipid hydroperoxidase activity of myoglobin and phenolic antioxidants in simulated gastric fluid. J. Agric. Food Chem., 53:3391-6.

Loguercio, C. and Di Pierro, M. 1999. The role of glutathione in the gastrointestinal tract: a review. Ital. J. Gastroenterol. Hepatol., 31:401-7.

Maerker, G., Nungesser, E.H. and Bunick, F. 1988. Reaction of cholesterol 5,6-epoxides with simulated gastric juice. Lipids, 23:761-65.

Marnett, L.J. 1999. Lipid peroxidation-DNA damage by malondialdehyde. Mutation Res., 424:83-95.

Márquez-Ruiz, G. and Dobarganes, M.C. 1992. Changes in endogenous lipid excretion in rats fed diets containing non-heated and thermally oxidized olive oils. Scand J. Gastroenterol., 27:1069-76.

Márquez-Ruiz, G. and Dobarganes, M.C. 1995. Assessments on the digestibility of oxidized compounds from [1-14C]-linoleic acid using a combination of chromatographic techniques. J. Chromatog. B., 675:1-8.

Márquez-Ruiz, G. and Dobarganes, M.C. 2006. Nutritional and physiological effects of used frying oils and fats. In: Erickson MD, ed. Deep Frying: Chemistry, Nutrition and Practical Applications. Champaign, IL: American Oil Chemists 'Society' 173-203.

Márquez-Ruiz, G., Guevel, G. and Dobarganes, M.C. 1998. Application of chromatographic techniques to evaluate enzymatic hydrolysis of oxidized and polymeric triglycerides by pancreatic lipase "in vitro". J. Am. Oil Chem. Soc., 75:119-26.

Márquez-Ruíz, G., Pérez-Camino, M.C. and Dobarganes, M.C. 1993. Evaluation of hydrolysis and absorption of thermally oxidized olive oil in non-absorbed lipids in the rat. Ann. Nutr. Metabol., 37:121-28.

Márquez-Ruiz, G., Pérez-Camino, M.C., Ruiz-Gutiérrez, V. et al. 1991. Absorción de grasas termoxidadas. I. Reproducibilidad y exactitud de las técnicas previas a la evaluación de lípidos no absorbidos. Grasas y Aceites, 42:32-7.

Márquez-Ruiz, G., Pérez-Camino, M.C. and Dobarganes, M.C. 1992. Digestibility of fatty acid monomers, dimers and polymers in the rat. J. Am. Oil Chem. Soc., 69:930-4.

Márquez-Ruiz, G., Pérez-Camino, M.C., Ruiz-Gutiérrez, V. et al. 1992. Absorción de grasas termoxidadas. II. Influencia del nivel de alteración y porcentaje de grasa en la dieta. Grasas y Aceites, 431:98-203.

Márquez-Ruiz, G., Pérez-Camino, M.C. and Dobarganes, M.C. 1992. In vitro action of pancreatic lipase on complex glycerides from thermally oxidized oils. Fat. Sci. Technol., 94:307-12.

Muller, C., Friedrichs, R., Wingler, K. et al. 2002. Perturbation of lipid metabolism by linoleic acid hydroperoxide in caco-2 cells. Biol. Chem., 383:637-48.

Nagatsugawa, K. and Kaneda, T. 1983. Absorption and metabolism of methyl linoleate hydroperoxides in rats. J. Jpn. Oil Chem. Soc., 32:362-66.

Penumetcha, M., Khan-Merchant, N. and Parthasarathy, S. 2000. Dietary oxidized fatty acids: an atherogenic risk? J. Lipid Res., 41:1473-80.

Penumetcha, M., Khan-Merchant, N. and Parthasarathy, S. 2002. Enhanced solubilization and intestinal absorption of cholesterol by oxidized linoleic acid. J. Lipid Res., 43:895-903.

Perkins, E.G., Endres, J.G. and Kummerow, F.A. 1961. The Metabolism of Fats I. Effect of dietary hydroxy acids and their triglycerides on growth, carcass, and fecal fat composition in the rat. J. Nutr., 73:291-8.
Piazza, G.J., Nunez, A. and Foglia, T.A. 2003. Isolation of unsaturated diols after oxidation of conjugated linoleic acid with peroxygenase. Lipids, 38:255-65

Reber, R.J. and Draper, H.H. 1970. Absorption and metabolism of 1-14Chydroxy octadecadienoate in the rat. Lipids, 5:983-7.

Rong, R., Ramachandran, S., Penumetcha, M. et al. 2002. Dietary oxidized fatty acids may enhance intestinal apolipoprotein A-I production. J. Lipid Res., 43:557-64.

Saguy, I.S. and Dana, D. 2003. Integrated approach to deep fat frying: engineering, nutrition, health and consumer aspects. J. Food Eng., 56:143-52.

Sánchez-Muniz, F.J., Arroyo, R., Sánchez-Montero, J.M. et al. 2000. In vitro digestibility study of thermal oxidised palm oleins. Food Sci. Technol. Int., 6:449-56.

Sánchez-Muniz, F.J., Bastida, S. and Gónzalez-Muñoz, M.J. 1999. Column and high-performance size exclusion chromatography applications to the in vivo digestibility study of a thermoxidized and polymerized olive oil. Lipids, 34:1187-92.

Sánchez-Muniz, F.J., Bastida, S., Márquez-Ruiz, G. et al. 2007. Effect of heating and frying on oil and food fatty acids. In: Chow, CK, ed. Fatty Acids in Foods and Their Health Implications . Boca Raton, FL: CRC Press. 511-43.

Sawa, T., Akaike, T., Kida, K. et al. 1998. Lipid peroxyl radicals from oxidized oils and heme-iron: Implication of a high fat diet in colon carcinogenesis. Cancer Epidemiol. Biomarkers Prev., 7:1007-12.

Spiteller, G. 2006. Peroxyl radicals:Induction of neurodegenerative and other inflammatory diseases. Their origin and how they transform cholesterol, phospholipids, plasmalogens, polyunsaturated fatty acids, sugars and proteins into deleterious products. Free Rad. Biol. Med., 41:362-87.

Staprans, I., Rapp, J., Pan, X.H. et al. 1994. Oxidized lipids in the diet are a source of oxidized lipids in chylomicrons of human serum. Arterioscler. Thromb., 14:1900-5.

Staprans, I., Hardman, D., Pan, X.H. et al. 1999. Effect of oxidized lipids in the diet on oxidized lipid levels in postprancial serum chylomicrons of diabetic patients. Diabetes Care, 22:300-6.

Staprans, I., Pan, X.M., Rapp, J.H. et al. 2000. Oxidized cholesterol in the diet accelerates the development of atherosclerosis in LDL receptorand apolipoprotein E-deficient mice. Arterioscler Thromb. Vasc. Biol., 20:708-14.

Stone, W.L. and Papas, A.M. 1997. Tocopherols and the etiology of colon cancer. J. Nat. Cancer Institute, 89:1006-14.

Suomela, J.P., Ahotupa, M. and Kallio, H. 2005. Triacylglycerol hydroperoxides not detected in pig small intestinal epithelial cells after a diet rich in oxidized triacylglycerols. Lipids, 40:349-53.

Suomela, J.P., Ahotupa, M., Sjovall, O. et al. 2004. Diet and lipoprotein oxidation: Analysis of oxidized triacylglycerols in pig lipoproteins. Lipids, 39:639-47.

Terao, J., Nagao, A., Yuki, H. et al. 1993. Reduction of fatty acid hydroperoxides by human parotid saliva. Lipids, $28: 121-4$.

Terao, J. and Fukino, N. 1993. Decomposition of phospholipid hydroperoxides in simulated gastric juice. J. Food Lipids, 1:79-86.

Tsunada, S., Iwakiri, R., Fujimoto, K. et al. 2003. Chronic lipid hydroperoxide stress suppresses mucosal proliferation in rat intestine: potentiation of ornithine decarboxylase activity by epidermal growth factor. Digest Dis. Sci., 48:2333-41.

Tsunada, S., Iwakiri, R., Noda, T. et al. 2003. Chronic exposure to subtoxic levels of peroxidized lipids suppresses mucosal cell turnover in rat small intestine and reversal by glutathione. Digest Dis. Sci., 48:210-22.

Ursini, F., Zamburlini, A., Cazzolato, G. et al. 1998. Posprandial plasma lipid hydroperoxides: a possible link between diet and atherosclerosis. Free Radic. Biol. Med., 25:250-2.

Velasco, J., Marmesat, S., Berdeaux, O. et al. 2004. Formation and evolution of monoepoxy fatty acids in thermoxidized olive and sunflower oils and quantitation in used frying oils from restaurants and fried food outlets. J. Agric. Food Chem., 52:4438-43. 
Velasco, J., Marmesat, S., Márquez-Ruiz, G. et al. 2004. Formation of short-chain fatty acids and oxidized monomeric triglycerides during deep-frying and occurrence in used frying fats. Eur. J. Lipid Sci. Technol., 106:728-35.

Vonk, R.J., Kalivianakis, M., Minich, D.M. et al. 1997. The metabolic importance of unabsorbed dietary lipids in the colon. Scand $J$. Gastroenterol., 32:65-7.

Wang, T.G., Gotoh, Y., Jennings, M.H. et al. 2000. Lipid hydroperoxideinduced apoptosis in human colonic $\mathrm{CaCo}-2$ cells is associated with an early loss of cellular redox balance. FASEB. J., 14:1567-76.
Wilson, R., Lyall, K., Smyth, L. et al. 2002a. Dietary hydroxy fatty acids are absorbed in humans: implications for the measurement of "oxidative stress" in vivo. Free Rad. Biol. Med., 32:162-8.

Wilson, R., Fernie, C.E., Scrimgeour, C.M. et al. 2002b. Dietary epoxy fatty acids are absorbed in healthy women. Eur. J. Clin. Invest, 32:79-83. 